\title{
LA COUR CONSTITUTIONNELLE DE LA FÉDÉRATION DE RUSSIE ET LES ÉVÉNEMENTS D'OCTOBRE 1993
}

Anne GAZIER, maître de conférences (HDR) de droit public, université Paris Ouest Nanterre La Défense; a.gazier@u-paris10.fr

RÉSUMÉ : La Cour constitutionnelle de la Fédération de Russie a, dans une décision en date du 21 septembre 1993, déclaré inconstitutionnel le décret $n^{\circ} 1400$, dans lequel Boris Eltsine prononçait la dissolution du Parlement russe, et affirmé que les actes du Président justifiaient sa destitution. Cette décision audacieuse, prise à une majorité de neuf voix contre quatre, a profondément divisé les juges. L'analyse du sténogramme de la réunion de la Cour montre que deux camps se sont affrontés et que les arguments échangés mêlaient étroitement considérations juridiques et politiques. À la suite de cette décision, la Cour a failli être supprimée. En effet, en octobre 1993, Boris Eltsine a suspendu les activités de la haute juridiction. La Conférence constitutionnelle a fortement envisagé de ne pas recréer de juridiction constitutionnelle à part entière dans le projet de nouvelle constitution. Finalement, si une Cour constitutionnelle a subsisté, c'est au prix d'un renforcement du rôle de l'exécutif dans le choix de ses juges et d'une réduction de ses attributions. Cela explique, au moins en partie, pourquoi la nouvelle Cour fait preuve, depuis sa mise en place en 1995, d'une très grande prudence sur toutes les questions concernant, de près ou de loin, le Président de la Fédération de Russie.

MOTS-CLÉS : Russie, Cour constitutionnelle, Boris Eltsine, 1993, politique, droit, justice, évènements d'octobre 1993 


\section{INTRODUCTION}

Le 21 septembre 1993 à 20 heures, Boris Nikolaïevitch Eltsine annonçait, dans une allocution télévisée, qu'il avait adopté un décret par lequel il prononçait la dissolution du Parlement de la Russie ${ }^{1}$. Ce décret, $\mathrm{n}^{\circ} 1400$, «sur la réforme constitutionnelle par étapes dans la Fédération de Russie » (Ukaz, 21.09.1993), prévoyait que « l'exercice des fonctions législative, administrative et de contrôle du Congrès des députés du peuple de la Fédération de Russie et du Soviet suprême de la Fédération de Russie [était] suspendu ». Il précisait également que jusqu'au début de l'activité du nouveau Parlement, le pays serait gouverné par des décrets du Président et des arrêtés du gouvernement. Le soir même, vers 22 heures, la Cour constitutionnelle de la Fédération de Russie s'est réunie. Dans une décision prise à une majorité de neuf juges contre quatre, elle a déclaré contraires à la Constitution l'allocution de Boris Eltsine et le décret $n^{\circ} 1400$ et a affirmé qu'ils justifiaient la destitution du Président (Zaključenie, 21.09.1993). Les événements tragiques des 3 et 4 octobre ont vu la prise d'assaut du Parlement et la victoire, par les armes, du camp présidentiel. Peu de temps après, le 6 octobre, le président de la Cour, Valerij Dmitrievič Zor'kin, a été contraint de démissionner. Dès le lendemain, le 7 octobre, Boris Eltsine a pris un décret suspendant les activités de la Cour (Ukaz, 07.10.1993). Avant d'analyser ces faits et de nous interroger sur l'attitude de la Cour constitutionnelle lors de cette crise politique et constitutionnelle majeure, un bref retour en arrière s'impose.

La création de la première Cour constitutionnelle de Russie remonte à une révision de la Constitution de la République socialiste fédéra-

1. Ce texte a pour origine une communication présentée lors du colloque « Un octobre oublié? La Russie en 1993 », qui s'est déroulé au Centre d'études et de recherches internationales (Ceri, Sciences Po) les 18 et 19 novembre 2013 avec le soutien du Centre d'étude des mondes russe, caucasien et centre-européen (Cercec, CNRS/EHESS), du Centre d'études franco-russe de Moscou (CEFR), de l'université Paris Ouest Nanterre La Défense, de l'Académie de l'économie et de la fonction publique de Russie (Ranhigs, Moscou), de la Fondation Maison des sciences de l'homme (FMSH, Paris), de l'Institut d'histoire russe de l'Académie des sciences de Russie (IRI RAN, Moscou), du laboratoire d'excellence Tepsis (Investissements d'avenir) et du ministère de l'Enseignement supérieur et de la Recherche (programme Access). tive soviétique de Russie (RSFSR) en date du 15 décembre 1990. Les attributions et le fonctionnement de la Cour ont été définis dans une loi du 12 juillet 1991 (Zakon, 1991). À la différence du Comité de surveillance constitutionnelle instauré en 1988 au niveau de l'URSS entière, la nouvelle Cour s'est vu reconnaître le pouvoir non seulement de se prononcer sur la constitutionnalité d'un grand nombre d'actes normatifs mais aussi d'annuler ceux-ci, ce qui en faisait une véritable juridiction constitutionnelle. Dans ces conditions, le choix des personnes appelées à faire partie de la Cour revêtait une très grande importance. Aussi le mode de désignation des juges a-t-il fait l'objet de dispositions détaillées dans la loi du 12 juillet 1991. Celle-ci fixait au préalable un certain nombre d'exigences qui pesaient sur les candidats à la fonction de juge constitutionnel : ils devaient avoir entre 35 et 60 ans, une expérience professionnelle dans le domaine juridique d'au moins dix ans, des « connaissances étendues dans le domaine du droit, un niveau élevé de qualification professionnelle, et les qualités morales indispensables à l'exécution de ses obligations par un juge de la Cour constitutionnelle de la RSFSR ». Les candidatures devaient être présentées soit par des groupes (ou « fractions ») de députés, soit par d'autres institutions (ministères, Prokuratura ${ }^{2}$, établissements d'enseignement, etc.). Ensuite, les membres de la Cour constitutionnelle devaient être élus par le Congrès des députés du peuple de la RSFSR. Plus précisément, elle instaurait une procédure de désignation des juges en trois temps : tout d'abord, un premier examen des candidatures par les commissions [komitety] du Soviet suprême de la RSFSR; ensuite, l'approbation de ces candidatures par le Soviet suprême; enfin, l'élection de chacun des juges, individuellement, par le Congrès des députés du peuple de la RSFSR, à la majorité des voix de l'ensemble des députés le composant.

Conformément à cette procédure, une vingtaine de candidatures ont été soumises au vote du Congrès fin octobre 1991. Seuls treize candidats ont obtenu la majorité nécessaire. Or, aux termes de la loi

2. On rappellera que la Prokuratura, dont la création remonte à Pierre Le Grand, est un organe de contrôle qui exerce une double fonction : une fonction de ministère public auprès des tribunaux et une fonction dite de « surveillance générale » de l'exécution des lois par différentes institutions - administrations, entreprises, associations, etc. 
du 12 juillet 1991, la Cour devait comporter quinze juges. Au lieu de reporter l'élection des deux juges manquants à une autre date, il a été décidé, comme la loi le permettait, de faire fonctionner la Cour avec seulement treize juges.

La composition de la Cour ainsi élue reflétait un savant équilibre entre les principales forces politiques du Soviet suprême (cinq juges ayant été présentés par des fractions communistes ou proches des communistes, deux par des fractions centristes et trois par des fractions réformatrices ${ }^{3}$ ). Elle faisait, par ailleurs, une place limitée à la représentation des minorités nationales (deux juges ${ }^{4}$ ) et des institutions judiciaires (un juge ayant été présenté par la Prokuratura ${ }^{5}$ ). Cette politisation était encore renforcée par le fait qu'une majorité de juges s'étaient engagés dans la vie politique - avant leur élection comme membres de la Cour -, en étant soit députés du peuple de la RSFSR (cinq juges ${ }^{6}$ ), soit député de la République autonome du Daghestan (un juge ${ }^{7}$ ) ou encore en s'étant présentés sans succès aux élections de 1990 (deux juges ${ }^{8}$ ). À la différence de l'engagement politique, l'expérience pratique dans le domaine juridique a été relativement peu prise en compte. En effet,

3. Comme l'indiquent notamment les biographies des juges (et anciens juges) de la Cour constitutionnelle figurant sur le site de la Cour, deux juges ont été présentés par la fraction Les communistes de Russie (Oleg Ivanovič Tiunov et Viktor Osipovič Lučin), un par le groupe de députés Les Communistes pour la démocratie (Valerij Dmitrievič Zor'kin), deux par la fraction La Russie, proche des communistes (Jurij Dmitrievič Rudkin et Nokolaj Trofimovič Vedernikov); deux juges ont été présentés par des fractions plutôt centristes : Nikolaj Vasil'evic Vitruk (fraction Changement-Nouvelle politique) et Tamara Georgievna

Morščakova (Les députés sans parti); trois ont été présentés par des fractions réformatrices : Ernest Mihajlovič Ametistov par la fraction Parti républicain de Russie-Parti social-démocrate de Russie, Anatolij Leonidovič Kononov par la fraction Russie démocratique. Ajoutons Vladimir Ivanovič Olejnik, présenté par le comité du Soviet suprême sur la liberté de conscience

et appartenant à plusieurs groupes politiques réformateurs. Voir le site de la Cour : <http://www.ksrf.ru/ru/Info/History/Pages/default.aspx>

4. Il s'agit du juge Boris Safarevič Ebzeev (représentant la République autonome de Karatchaevo-Tcherkessie) et du juge Gadis Abdullaevič Gadžiev (représentant la République autonome du Daghestan).

5. Il s'agit du juge Nikolaj Vasil'evič Seleznëv.

6. Il s'agit des juges A. L. Kononov, V. I. Olejnik, Ju. D. Rudkin, O. I. Tiunov

et N. T. Vedernikov.

7. Il s'agit du juge G. A. Gadžiev.

8. Il s'agit des juges E. M. Ametistov et V. D. Zor'kin. sur les treize juges, seuls trois étaient des juristes praticiens (ils avaient travaillé dans la Prokuratura ${ }^{9}$ ); les dix autres étaient des professeurs de droit ayant exercé dans des établissements d'enseignement supérieur.

Composition de la Cour constitutionnelle de la Fédération de Russie élue en 1991

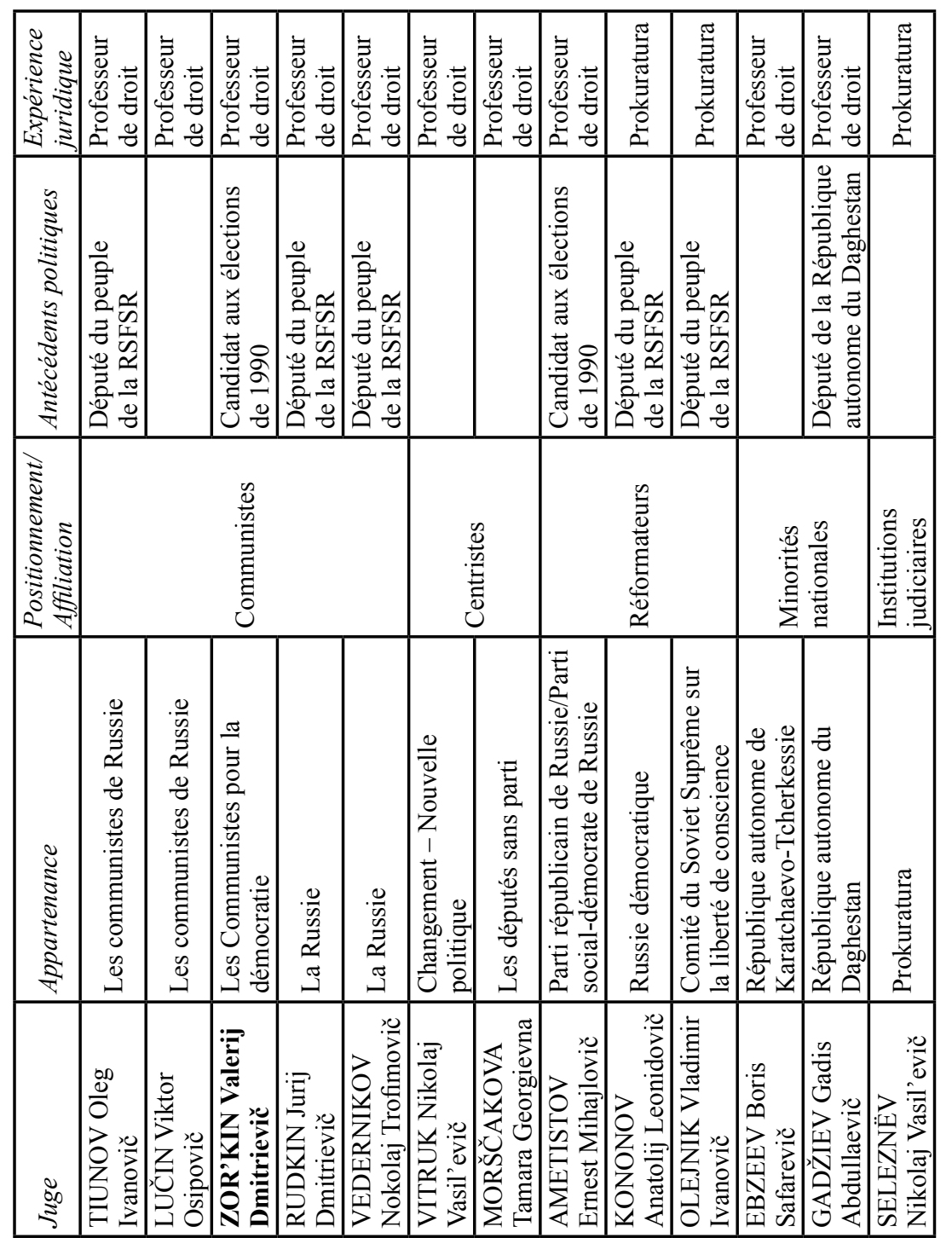

9. Il s'agit des juges A. L. Kononov, V. I. Olejnik et N. V. Seleznëv. 
Les juges ainsi désignés étaient soumis, aux termes de la loi du 12 juillet 1991, à une stricte obligation de neutralité. Ils devaient notamment se tenir à l'écart de la vie politique (ne pas appartenir à un parti politique, ne pas participer à des actions politiques, ne pas être député, etc.). Une liste importante d'incompatibilités était destinée à leur permettre de n'être soumis qu'à la Constitution. En outre, la loi précisait explicitement que la Cour, chargée d'exercer la justice constitutionnelle, «n'examine pas les questions politiques » (art. $\left.1^{\mathrm{er}}, \S 3\right)$.

La Cour, qui a commencé à fonctionner fin 1991, après l'élection, par les juges, de Valerij Zor'kin comme président, a-t-elle respecté ces exigences? Dès ses premiers pas, elle a fait preuve d'audace à l'égard du pouvoir politique et, notamment, du Président de la Russie. Elle a, en effet, annulé, dans le premier arrêt qu'elle a adopté, début 1992, un décret présidentiel (qui organisait la fusion des organes de la sécurité d'État et de l'Intérieur) de Boris Eltsine (Postanovlenie, 14.01.1992). Peu après, elle s'est montrée aussi exigeante à l'égard $\mathrm{du}$ Parlement, censurant plusieurs de ses actes (Konstitucionnyj sud. Epoha Zor'kina, 1993). Le 30 novembre 1992, après avoir examiné pendant plusieurs mois ce que l'on a appelé «l'affaire du Parti communiste de l'Union soviétique (PCUS) », c'est-à-dire la question de la constitutionnalité des décrets pris, à la suite de l'échec du putsch d'août 1991, par Boris Eltsine pour interdire les activités du PCUS et du parti communiste de la RSFSR, elle a rendu un arrêt de compromis : il reconnaissait la plupart des dispositions qui étaient attaquées comme conformes à la Constitution, mais pas toutes; en particulier, la dissolution de certaines organisations de base du Parti a été considérée comme contraire au texte constitutionnel (Postanovlenie, 30.11.1992).

Petit à petit, cependant, ce souci d'impartialité, ou tout au moins cette recherche d'une position équilibrée entre les forces politiques du pays, a fait place à un net engagement en faveur du Parlement dans le conflit de plus en plus aigu qui l'a opposé au Président de la Fédération de Russie $^{10}$. Loin de se tenir à l'écart de la bataille que se livraient

10. Lire notamment, sur cette évolution du rôle de la Cour constitutionnelle russe : BAUDOUIN, 2005, 2010; EPSTEIN, KNIGHT \& SHEVTSOVA, 2001; TROCHEV, 2008

Volume 47, septembre 2016 les deux pouvoirs, en effet, la Cour et, surtout son président, Valerij Zor'kin, ont cherché à jouer un rôle de médiateur tout en soutenant ouvertement le camp de l'organe législatif. Valerij Zor'kin notamment, sortant de la réserve requise pour un juge constitutionnel, a multiplié les interventions critiques à l'égard du Président dans les médias ${ }^{11}$. La jurisprudence de la Cour a porté la marque de cette orientation politique. Ainsi, la haute juridiction a annulé plusieurs décrets adoptés par Boris Eltsine ${ }^{12}$ ainsi que sa décision, annoncée à la télévision le 20 mars $1993^{13}$, de renforcer les pouvoirs de l'exécutif en instaurant un « mode spécial d'administration $\gg$ dans le pays ${ }^{14}$.

Toutefois, cette attitude de la Cour ne faisait pas l'unanimité parmi ses membres. Bien au contraire, les juges se sont montrés de plus en plus divisés. Peu à peu s'est formé un groupe de quatre juges (comprenant Ernest Mihajlovič Ametistov, Anatolij Leonidovič Kononov, Tamara Georgievna Morščakova et Nikolaj Vasil'evič Vitruk) qui ont voté, à compter de 1993, contre les décisions de la Cour censurant un acte du Président et émis des opinions dissidentes à leur encontre ${ }^{15}$. L'un de ces

11. Le rôle - ouvertement politique - joué par Valerij Zor'kin à cette époque est retracé dans de nombreuses études. (Lire notamment GÉLARD, 1993 et le document précité Konstitucionnyj sud. Epoha Zor'kina, 1993.)

12. Les arrêts censurant les décrets présidentiels étaient assez divers. On citera, par exemple, celui, en date du 12 février 1993, qui a censuré un décret prononçan la dissolution des structures d'une organisation politique, le Front de salut national (Postanovlenie, 12.02.1993).

13. Au cours de cette intervention télévisée, Boris Eltsine a annoncé l'adoption d'un décret introduisant un « mode spécial d'administration du pays » [osobyj porjadok upravelija stranoj] jusqu'à la fin de la crise constitutionnelle. Il s'agissait d'un renforcement des pouvoirs de l'exécutif et, notamment, de ceux du Président. Celui-ci s'est vu, en particulier, reconnaître le droit de prendre « toutes les mesures indispensables à la défense de l'ordre constitutionnel ». Le décret prévoyait également qu'étaient dépourvues de force juridique les décisions des organes et des dirigeants destinées à suspendre les actes du Président (Ukaz, 20.03.1993).

14. La Cour constitutionnelle a déclaré contraires à la Constitution non seulement le décret introduisant un " mode spécial d'administration » mais aussi la déclaration faite par Boris Eltsine à la télévision (Zaključenie, 23.03.1993; Konstitucionnyj sud. Epoha Zor'kina, 1993).

15. Lire, à ce sujet, la liste des opinions dissidentes de la Cour constitutionnelle, publiée sous la forme d'un tableau [tablica osobyh mennij sudej Konstitucionnogo Suda RF (čast' 1)] par l'Institut du Précédent [Institut 
quatre juges, Nikolaj Vitruk, occupait le poste de vice-président de la Cour. Ainsi, la direction de la haute juridiction était divisée entre d'un côté le président Zor'kin, actif défenseur du Parlement, et de l'autre le vice-président Vitruk, partisan de Boris Eltsine.

C'est cette Cour très politisée et divisée qui a été amenée à examiner le 21 septembre 1993 la constitutionnalité du décret $n^{\circ} 1400$ par lequel Boris Eltsine prononçait la dissolution du Parlement. La décision de censure qu'elle a alors adoptée a eu des incidences multiples, qualifiées de « dramatiques » sur le site actuel de cette juridiction ${ }^{16}$. Avant de les analyser, il faut nous interroger sur les motifs qui sont à l'origine de l'adoption d'une telle décision. Nous disposons pour ce faire de plusieurs sources et, d'abord, du sténogramme de la réunion de la Cour pendant laquelle a été prise la décision, le 21 septembre $1993^{17}$. Certes, il est incomplet, mais l'essentiel des arguments échangés par les juges y figure. Publié en 2003 par le journal Moskovskie Novosti, il n'a, à notre connaissance, pas encore fait l'objet d'une véritable analyse. Les autres sources sont, outre le texte de la décision et celui des opinions dissidentes des quatre juges qui ont voté contre ${ }^{18}$, divers entretiens accordés par la suite par les membres de la Cour ainsi que les articles et mémoires écrits par certains d'entre eux.

Il apparaît à la lecture de ces textes et témoignages que la décision de la Cour du 21 septembre 1993 a été prise au terme d'un vif affrontement aussi bien juridique que politique et qu'elle a eu des implications tout à fait importantes, à court comme à long terme.

Precedenta] à l'adresse : http://precedent.eu/ru/index.php?id=39 (consulté le 26.08.2015)

16. Voir le site de la Cour : http://www.ksrf.ru/ru/Info/History/Pages/default.aspx (consulté le 24.08.2015).

17. On peut lire ce sténogramme - malheureusement incomplet - sur Internet (il a été publié, pour la première fois, en 2003, par le journal Moskovskie Novost qui a réussi à se le procurer) à l'adresse suivante : http://www.garweb.ru/conf/ ks/20031126/smi/msg.asp@id_msg315019.htm (consulté le 24.08.2015).

18. Lire la décision [zaključenie] précitée du 21 septembre 1993 et les quatre opinions dissidentes qui sont publiées à sa suite dans le bulletin officiel

de la Cour constitutionnelle : Vestnik Konstutionnogo Suda Rossijskoj Federacii, $\mathrm{n}^{\circ} 6,1994$, p. $40-56$

\section{UNE DÉCISION MÊLANT ÉTROITEMENT DROIT ET POLITIQUE}

Il convient au préalable de s'interroger sur la façon dont s'est déroulée la réunion du 21 septembre 1993. Elle a eu lieu à la Cour constitutionnelle, à l'initiative de son président, Valerij Zor'kin (sans respecter le décret $n^{\circ} 1400$ qui invitait la Cour à suspendre ses audiences ${ }^{19}$ ). Elle s'est tenue sur le fondement du droit de la Cour de se saisir elle-même. Tous les juges s'accordent sur un point : cette audience (qui a pris la forme d'une simple réunion, sans que les juges aient revêtu leur robe) a été marquée par la précipitation et la violation des règles élémentaires de procédure - absence d'examen préalable de l'affaire, d'information et de représentation des parties, motivation pour le moins succincte de la décision, etc. Mais, selon les juges qui ont approuvé la décision, cette précipitation était justifiée par l'urgence ${ }^{20}$

Pour le reste, deux camps apparaissent nettement : les partisans de la décision prise par la Cour le 21 septembre; les adversaires de cette décision. Seul un juge (Nikolaj Vasil'evič Seleznëv) a exprimé son refus de prendre position sur la question de la constitutionnalité du décret $\mathrm{n}^{\circ} 1400$ (en dénonçant l'irrégularité de la réunion de la Cour) mais il a ensuite pris position en votant pour la décision de la Cour ${ }^{21}$. Quels ont été les arguments des uns et des autres?

\section{LES PARTISANS DE LA DÉCISION : LA DOMINATION DES ARGUMENTS JURIDIQUES}

Si on se réfère au sténogramme de la réunion, les juges qui ont voté pour la décision se sont placés essentiellement sur le terrain du droit. Cinq d'entre eux ${ }^{22}$ se sont contentés d'affirmer que le décret $n^{\circ} 1400$ était contraire à la Constitution (notamment parce qu'aucune disposi-

19. Lire le point 10 du décret précité, $\mathrm{n}^{\circ} 1400$, qui est ainsi rédigé : « proposer à la Cour constitutionnelle de la Fédération de Russie de ne pas convoquer d'audience jusqu'au début du fonctionnement de l'Assemblée fédérale de la Fédération de Russie ».

20. Lire notamment l'entretien avec Nikolaj Vedernikov (Političeskij Žurnal, 2004).

21. Voir le sténogramme de la réunion de la Cour cité plus haut. 22. Il s'agit de Gadis Gadžiev, Vladimir Olejnik, Jurij Rudkin, Oleg Tiunov et Nikolaj Vedernikov. 
tion constitutionnelle ne donnait au Président de la Fédération de Russie le droit de dissoudre le Congrès des députés du peuple et le Soviet suprême). Cependant, trois juges ont utilisé des arguments plus politiques. Ainsi, Boris Safarovič Ebzeev a mis en cause le rôle joué par le Président dans l'aggravation de la situation politique; Viktor Osipovič Lučin a dénoncé "l'hypocrisie monstrueuse » de l'auteur du décret $\mathrm{n}^{\circ} 1400$; Valerij Zor'kin a stigmatisé « l'usurpation de tout le pouvoir au sein de l'État » par le Président et la réalisation d'un « coup d'État ». Observons que si les juges de ce « camp » avaient le droit pour eux, certains d'entre eux, et surtout le président Zor'kin, étaient, comme nous l'avons déjà vu, loin d'être neutres et soutenaient ouvertement le Soviet suprême. De même, les neuf juges qui ont voté pour la décision du 21 septembre n'en étaient pas à leur première censure des décisions de Boris Eltsine. Ils avaient, en particulier, voté pour la décision de la Cour du 23 mars 1993 qui avait reconnu le caractère inconstitutionnel de l'intervention télévisée de Boris Eltsine annonçant l'instauration d'un « mode spécial d'administration » dans le pays. Certes, l'alignement des juges sur les positions du Soviet suprême dans le conflit qui l'a opposé au Président n'était pas total. Rappelons notamment que Valerij Zor'kin était partisan non pas de la « république parlementaire » souhaitée par le pouvoir législatif mais de la « république présidentielle » prônée par l'exécutif ${ }^{23}$. Il est difficile, cependant, d'affirmer avec lui que la décision du 21 septembre 1993 aurait été prise non pas pour apporter un soutien au Parlement mais sur une base exclusivement juridique ${ }^{24}$.

\section{LES ADVERSAIRES DE LA DECISION : LA PREPONDERANCE DES CONSIDÉRATIONS POLITIQUES}

Toujours en se référant au sténogramme de la réunion, on constate que les arguments des juges hostiles à la décision ont présenté un caractère nettement politique. Anatolij Kononov et Tamara Morščakova, notamment, ont reconnu que d'un point de vue strictement juridique, le décret $n^{\circ} 1400$ était contraire à la Constitution mais ils ont affirmé qu'il fallait tenir compte d'autres considérations. Anatolij Kononov, notamment, a fait valoir que le pays était dans une situation d'impasse, de confrontation entre deux pouvoirs, qui ne laissait pas d'autre issue possible tandis

23. Lire l'entretien avec Valerij Zor'kin (GAMOV, 2003).

24. Lire l'entretien avec Valerij Zor'kin (SUHOVA \& ZAMAHIN, 2003). que Tamara Morščakova a déclaré que le Soviet suprême et le Président violaient l'un et l'autre la Constitution et qu'il était impossible d'exiger d'une partie ce que l'on ne pouvait exiger de l'autre. Elle a proposé que la Cour démissionne. Nikolaj Vitruk, le vice-président de la Cour, a, quant à lui, affirmé son plein accord avec Boris Eltsine et la façon dont il avait décrit, dans son allocution, la situation de la Russie. Enfin, Ernest Ametistov a surtout plaidé pour l'organisation d'une rencontre entre les juges de la Cour et le Président de la Russie.

Dans leurs opinions dissidentes ${ }^{25}$, les quatre juges ont considérablement développé leurs arguments. Sur la forme, ils ont contesté les bases juridiques sur lesquelles a été prise la décision de la Cour. Ils ont estimé, en particulier, que si le pouvoir d'auto-saisine utilisé par la haute juridiction lui permettait de se prononcer sur la constitutionnalité des agissements et des décisions individuelles du Président, il ne pouvait être employé pour censurer un acte normatif (en l'occurrence, le décret $\left.\mathrm{n}^{\circ} 1400\right)$. Sur le fond, ils ont confirmé que d'un point de vue strictement formel, ce décret comprenait des dispositions allant au-delà de ce que permettait la Constitution. Mais ils ont fait valoir qu'il était indispensable de prendre en compte le contexte de l'adoption de ce texte et notamment :

- la nécessité de respecter la volonté, exprimée par plus de la moitié des votants lors du référendum du 25 avril 1993 de voir des élections législatives anticipées être organisées, $63,7 \%$ des votants s'étant prononcés pour la tenue de ces élections (E. M. Ametistov, T. G. Morščakova). On rappellera que ce pourcentage n'avait pas été reconnu suffisant car seul comptait, en vertu d'une décision de la Cour constitutionnelle (Postanovlenie, 21.04.1993) le pourcentage des électeurs inscrits (qui était de 43,1\%);

- l'activité « contraire au droit et aux intérêts du peuple » du Congrès des députés du peuple et du Soviet suprême, considérée comme menaçan l'intégrité et la souveraineté de la Russie (E. M. Ametistov);

- la situation de «nécessité extrême » (A. L. Kononov) dans laquelle se trouvait le Président, qui l'a poussé à tout faire pour sortir d'une situation de paralysie du pouvoir;

- l'insuffisance des pouvoirs reconnus au Président par la Constitution.

25. Lire les quatre opinions dissidentes (Zaključenie, 21.09.1993). 
On le voit, ces arguments traduisent un net engagement en faveur du Président de la Russie. D'ailleurs, comme nous l'avons déjà mentionné, les quatre juges concernés formaient un groupe qui s'opposait à la ligne favorable au Parlement adoptée par la Cour à compter de 1993. Ils étaient même considérés à l'époque comme des partisans de Boris Eltsine. Notamment, trois d'entre eux avaient voté contre la décision du 23 mars 1993 censurant l'instauration d'un mode spécial d'administration $^{26}$ et les quatre avaient fait partie des six juges constitutionnels rencontrés par le Président russe en mai $1993^{27}$.

C'est ainsi dans un contexte conflictuel que la Cour constitutionnelle a adopté sa décision. D'ailleurs, comme nous l'avons signalé plus haut, la Cour ne s'est pas contentée de déclarer contraires à la Constitution l'allocution d'Eltsine et le décret $n^{\circ} 1400$. Sous l'impulsion de son président, Valerij Zor'kin, elle a estimé que ces actes justifiaient la destitution de Boris Eltsine. Comme nous le verrons plus loin, le sténogramme de la réunion indique que Valerij Zor'kin a soumis à deux reprises son projet de décision au vote et n'a obtenu la majorité que lors du deuxième vote. Certains, comme Ernest Ametistov, affirment qu'il y aurait eu un truquage des résultats (Ametistov, 1999-2001). Rien ne permet aujourd'hui d'affirmer que de telles irrégularités ont été commises.

Ce qui est avéré, c'est que les juges se sont divisés sur les fondements juridiques de la décision de la Cour ou, plus précisément, sur le point de savoir sur quel article de la Constitution elle pouvait se baser pour déclarer que les actes commis par Eltsine justifiaient sa destitution. En effet, la Constitution de la Fédération de Russie de 1978, dans sa dernière rédaction (datant des 9 et 10 décembre 1992), comportait deux articles distincts sur la possibilité de mettre fin aux fonctions du Président de la Russie : les articles $121-6$ et $121-10$. Or tous les deux posaient des problèmes d'utilisation à la Cour. Selon les termes du premier d'entre eux (121-6) :

"Les pouvoirs du Président de la Fédération de Russie ne peuvent pas être utilisés pour changer la structure étatique nationale de la Fédération

26. Il s'agit d'Ernest Ametistov, de Tamara Morščakova et de Nikolaj Vitruk Lire notamment le récit précité Konstitucionnyj sud. Epoha Zor'kina, 1993. 27. Ibid. de Russie, pour dissoudre tout organe du pouvoir d'État élu régulièrement ou suspendre son activité; dans le cas contraire, ils cessent immédiatement. »

Cet article présentait un inconvénient majeur : il ne précisait pas du tout la procédure à suivre pour que les pouvoirs du Président « cessent immédiatement ». Cette lacune le rendait difficile à employer. Le deuxième article (121-10) soulevait moins de difficultés. Il dispose que :

«Le Président de la Fédération de Russie peut être destitué en cas de violation de la Constitution de la Fédération de Russie, des lois de la Fédération de Russie ainsi que du serment qu'il a prêté. Cette décision est prise par le Congrès des députés du peuple de la Fédération de Russie sur la base d'une décision [zaključenie] de la Cour constitutionnelle de la Fédération de Russie, à la majorité des deux tiers des voix de l'ensemble des députés du peuple de la Fédération de Russie, à l'initiative du Congrès des députés du peuple de la Fédération de Russie, du Soviet suprême de la Fédération de Russie ou de l'une de ses Chambres. »

Ainsi, cet article, qui traite de la destitution du Président proprement dite, prévoit l'intervention de la Cour constitutionnelle avant le vote sur la destitution par le Congrès. Mais il précise aussi que la procédure doit être initiée par le Parlement (Congrès ou Soviet suprême). Or lorsque la Cour constitutionnelle s'est réunie le 21 septembre 1993 au soir, une telle initiative n'avait pas été prise, tout au moins officiellement. Ainsi, aucun de ces deux articles ne pouvait donner un fondement incontestable à la partie de la décision de la Cour affirmant que les actes commis par Boris Eltsine justifiaient sa destitution, l'article 121-6 étant particulièrement inadapté. Pourtant, Valerij Zor'kin a tenu à ce que la décision de la Cour s'appuie sur ces deux articles (pour lui donner plus de poids, semble-t-il). Lors d'un premier vote, cinq juges ont donné leur accord à cette solution, quatre (Boris Ebzeev, Gadis Abdullaevič Gadžiev, Jurij Dmitrievič Rudkin et Nikolaj Seleznëv) ont voté « pour» tout en s'opposant à l'utilisation de l'article 121-6 et quatre ont voté « contre ». Lors d'un deuxième vote, les quatre juges qui étaient hostiles à l'emploi de l'article 121-6 ont finalement voté pour la décision que souhaitait le président de la Cour. C'est ainsi que la majorité a été atteinte : neuf voix pour, quatre contre. 
Cette division des juges explique probablement - en plus de l'urgence -, pourquoi la motivation de la décision de la Cour est si courte ${ }^{28}$ (ce qui est inhabituel et contraste, notamment, avec la décision précitée du 23 mars 1993 sur la déclaration de Boris Eltsine annonçant l'introduction d'un mode spécial d'administration ${ }^{29}$ ).

Une fois la décision adoptée, Valerij Zor'kin est allé à la «Maison Blanche », le siège du Parlement russe, et le lendemain, le Soviet suprême, se fondant sur l'article 121-6 de la Constitution, a prononcé la cessation des fonctions du Président de la Fédération de Russie et son remplacement par le vice-président, Aleksandr Vladimirovič Ruckoj.

\section{UNE DÉCISION AUX CONSÉQUENCES « DRAMATIQUES »?}

La décision prise par la Cour le 21 septembre 1993 a-t-elle eu des incidences importantes sur les événements d'octobre? Cette question appelle une réponse nuancée. On observera d'abord que les juges de la Cour sont, plusieurs années après, en désaccord sur ce point. Certains, comme Ernest Ametistov (qui avait voté contre la décision), ont estimé qu'elle avait servi de "fondement juridique à tout ce qui s'était passé ensuite et s'était conclu par la tragédie des 3 et 4 octobre » (Toročešnikova, 2002). D'autres, comme Gadis Gadžiev (qui avait voté pour) ont fait valoir qu'il est impossible «d'affirmer que c'est précisément la position de la Cour qui a provoqué l'effusion de sang ». Ce

28. En effet, la partie de la décision de la Cour qui contient la motivation comprend seulement une dizaine de lignes. C'est la suivante : « Le décret du Président de la Fédération de Russie B. N. Eltsine "Sur la réforme constitutionnelle par étapes dans la Fédération de Russie" du 21 septembre 1993 $n^{\circ} 1400$ et sa déclaration aux citoyens de la Russie du 21 septembre 1993 ne sont pas conformes à la deuxième partie de l'article 1 , à la deuxième partie de l'article 2 , à l'article 3 , à la deuxième partie de l'article 4 , aux première et troisième parties de l'article 104, à la troisième partie du point 11 de l'article 121-5, à l'article 121-6, à la deuxième partie de l'article 121-8, aux articles 165-1, 177 de la Constitution de la Fédération de Russie et servent de fondement à la destitution du Président de la Fédération de Russie B. N. Eltsine ou à la mise en œuvre d'autres mécanismes spéciaux de sa responsabilité selon les modalités prévues aux articles 121-10 ou 121-6 de la Constitution de la Fédération de Russie ». Voir Zaključenie, 21.09.1993.

29. Voir la motivation de cette décision : Zaključenie, 23.03.1993. serait « exagérer manifestement le rôle de la Cour comme le rôle de la Constitution $»^{30}$.

Ensuite, il convient de remarquer que l'impact de la décision du 21 septembre sur le cours des évènements a été limité par le rôle joué par la Cour ou, plutôt, par son président, juste après son adoption. Ainsi, Valerij Zor'kin, qui est en grande partie à l'origine de cette décision, n'a pas ménagé ses efforts dans les jours qui ont suivi pour tenter une médiation entre le pouvoir législatif et le pouvoir exécutif. Dès le 22 septembre, il a adopté une déclaration dans laquelle il préconisait la tenue d'élections présidentielle et législatives anticipées (Zajavlenie, 22.09.1993). Puis il a organisé toute une série de réunions avec des représentants des régions pour soutenir ce qui a été appelé la « variante nulle » (c'est-à-dire l'organisation d'élections anticipées du Parlement et du Président ainsi que l'annulation des actes adoptés par les deux branches du pouvoir à compter du 21 septembre $)^{31}$. Certes, ce faisant, Valerij Zor'kin prenait une part active à la vie politique. D'ailleurs deux juges (Ernest Ametistov et Nikolaj Vitruk) ont décidé de ne plus participer aux audiences de la haute juridiction en guise de protestation ${ }^{32}$. Il n'en reste pas moins vrai qu'en prenant ces initiatives, le président de la Cour s'efforçait de concilier les parties et non de les dresser l'une contre l'autre.

Face à l'échec de cette stratégie et aux affrontements violents des 3 et 4 octobre, la Cour constitutionnelle a affirmé, le 5 octobre, qu'elle n'était plus en état de juger la constitutionnalité des traités et des actes normatifs mais qu'elle continuerait à examiner les affaires relatives aux actes individuels (Zajavlenie, 05.10.1993). En définitive, si la décision du 21 septembre a entrainé des conséquences « dramatiques », ne seraitce pas plutôt pour la Cour elle-même? Il apparait, en effet, qu'elle a failli disparaître et qu'elle a subi d'importantes transformations pour pouvoir fonctionner à nouveau.

30. Lire l'entretien avec Gadis Gadžiev (ZAKATNOVA, 2001)

31. Lire à ce sujet Konstitucionnyj sud. Epoha Zor'kina, 1993, déjà cité, et la « demande » tout à fait officielle faite par Valerij Zor'kin le 28 septembre 1993 (Trebovanie, 28.09.1993).

32. Lire le récit précité : Konstitucionnyj sud. Epoha Zor’kina, 1993. 


\section{LES MENACES PESANT SUR L'EXISTENCE DE LA COUR}

Comme nous l'avons dit dans l'introduction, un décret pris par Boris Eltsine le 7 octobre 1993 a suspendu les activités de la Cour. Plus exactement, il lui a interdit de tenir des audiences « jusqu'à l'adoption de la nouvelle Constitution de la Fédération de Russie $»^{33}$. C'est une décision grave mais on aurait pu s'attendre à une mesure plus radicale. Dans le décret précité, Boris Eltsine a condamné très vivement l'attitude de la Cour, qui était devenue, selon lui, « un instrument de la lutte politique présentant un danger exceptionnel pour l'État $»^{34}$, mais il ne l'a pas supprimée pour autant. Il n'a pas mis fin non plus aux fonctions des juges constitutionnels. Ces derniers ont pu continuer à se réunir, notamment, pour examiner le projet de Constitution et celui de nouvelle loi relative à la Cour constitutionnelle. La seule chose qu'ils ne pouvaient plus faire, c'était exercer leur fonction de juge. Comment, tout de même, expliquer que la Cour n'ait pas été purement et simplement supprimée? Nikolaj Vitruk, qui était à l'époque vice-président de la Cour et qui avait voté contre la décision du 21 septembre, a affirmé que la suppression de la Cour avait bel et bien été envisagée par le pouvoir exécutif mais que la démission - contrainte et forcée - du président de la Cour, Valerij Zor'kin, le 6 octobre, avait permis d'éviter qu'une telle mesure soit prise. Le chef de l'administration du Président de l'époque, Sergej Aleksandrovič Filatov, a confirmé cette version des faits, affirmant qu'il avait même eu entre ses mains le texte d'un décret présidentiel supprimant la Cour constitutionnelle et qu'il n'avait pu le déchirer qu'en échange de la «démission» de V. Zor'kin (Ogonëk, 2005). Nikolaj Vitruk, quant à lui, a mis en avant le rôle qu'il a joué lui-même à ce moment, grâce notamment aux relations qu'il entretenait avec des membres de l'administration présidentielle. Il s'est presque posé en sauveur de la Cour (Vitruk, 2001).

Par la suite, lorsqu'ont repris les travaux sur le projet de nouvelle Constitution, la question du maintien ou non d'une Cour constitutionnelle - qui avait déjà fait l'objet de débats au sein de la Conférence constitutionnelle [Konstitucionnoe soveščanie] - a pris une importance

33. Lire le décret précité du 7 octobre 1993, et tout particulièrement son premier point (Ukaz, 07.10.1993).

34. Lire le cinquième paragraphe du décret précité. toute nouvelle ${ }^{35}$. En effet, avant octobre 1993, la nécessité pour la Russie d'avoir une telle Cour était reconnue par la plupart des participants à cette Conférence mais après ces évènements, l'opportunité d'avoir une telle juridiction - qui s'était engagée dans la bataille politique - a été très discutée ${ }^{36}$. Notamment, il a été fortement envisagé de ne pas créer de juridiction constitutionnelle spécialisée mais d'instaurer un « arbitrage constitutionnel » ou une simple chambre constitutionnelle au sein de la Cour suprême, avec très peu de pouvoirs. Selon Nikolaj Vitruk, devenu président de la Cour par intérim, les juges constitutionnels, qui ont eu à examiner ces propositions, se sont battus pour l'existence d'une véritable Cour constitutionnelle. Il a insisté aussi sur le rôle de tout premier plan joué par la Commission de Venise du Conseil de l'Europe à cet égard ${ }^{37}$. En définitive, ce qui semble avoir emporté l'adhésion du pouvoir exécutif, c'est l'idée selon laquelle il était possible de conserver une Cour constitutionnelle tout en la dépolitisant.

\section{LA MISE EN PLACE D’UNE COUR PLUS « DOCILE »}

Afin d'éloigner la Cour de la politique, un certain nombre de changements ont été apportés tant à sa composition qu'à ses attributions, grâce à la Constitution de 1993 et à la nouvelle loi sur la Cour constitutionnelle du 21 juillet 1994

Concernant la composition de la nouvelle Cour, le mode de désignation des juges a changé. Il a été prévu, en effet, que les juges ne seraient plus élus par le Parlement comme auparavant mais désignés par la seule Chambre haute (le Conseil de la Fédération ${ }^{38}$ ) sur proposi-

35. On rappellera que cette Conférence constitutionnelle, créée à l'initiative du Président de la Fédération de Russie, s'est réunie du 29 avril au 10 novembre 1993, donc avant et après les évènements d'octobre 1993.

36. Lire notamment MITJUKOV, 2014, p. 43-50.

37. Lire VITRUK, 2001. On précisera que la Commission européenne pour la démocratie par le droit - dite « Commission de Venise » est un organe consultatif du Conseil de l'Europe sur les questions constitutionnelles. Cette Commission a donné son avis à de nombreuses reprises, durant les années 1992-1993, sur les différents projets de Constitution russe. Elle s'est toujours montrée très attentive au rôle de la Cour constitutionnelle et aux garanties de son indépendance; lire notamment l'introduction de l'avis qu'elle a rendu sur la Constitution de la Fédération de Russie du 12 décembre 1993 (Commission de Venise, 1994). 38. On précisera que le Conseil de la Fédération a été élu au suffrage 
tion du Président de la Russie. Il s'agissait, pour réduire l'influence des partis et des groupes politiques, de ne plus confier le choix des juges à une assemblée représentant le peuple et d'instaurer une procédure associant le pouvoir législatif et le pouvoir exécutif. D'importantes discussions ont ensuite eu lieu sur la question de savoir quel sort il convenait de réserver aux juges de l'ancienne Cour : devaient-ils faire partie de la nouvelle Cour ou bien quitter leurs fonctions et céder la place à des juges élus selon la nouvelle procédure? Lors des réunions de la Conférence constitutionnelle, les partisans d'un renouvellement total des juges - parmi lesquels les juges constitutionnels Nikolaj Vitruk et Tamara Morščakova - ont fait valoir, en particulier, que les compétences de la nouvelle Cour et le nouveau mode de désignation des juges justifiaient un tel renouvellement. À l'inverse, ceux qui souhaitaient le maintien en fonctions des juges de l'ancienne Cour ont mis en avant le principe de l'inamovibilité des juges et ont affirmé que le respect de ce principe s'impose, dans un État de droit, même en cas de changement des pouvoirs d'une juridiction. Ce dernier point de vue, partagé notamment par le représentant de l'administration présidentielle, Sergej Filatov, est celui qui a finalement prévalu ${ }^{39}$. C'est ainsi qu'il a été décidé que les treize membres de l'ancienne Cour resteraient en poste. Pour permettre néanmoins le recrutement de nouveaux juges, le nombre total de membres de la Cour a été augmenté. Il a été porté à dix-neuf juges. Selon certains et notamment le juge Gadis Gadžiev, ce chiffre de dixneuf n'est pas du tout le fruit du hasard : il s'agissait, pour le Président, de s'assurer une majorité au sein de la Cour, en faisant en sorte que les neuf juges qui avaient voté pour la décision du 21 septembre aient face à eux dix juges partisans du Président : les quatre qui avaient voté contre la décision en question et six qui seraient désignés selon la nouvelle procédure, sur proposition du Président (Višnevskij, 2011).

S'agissant des attributions de la Cour, on lui a, d'abord et avant tout, enlevé le droit de se saisir elle-même. Ce pouvoir a été considéré, en effet, comme ayant été à l'origine de la décision du 21 septembre et comme ayant incité, plus généralement, la Cour à se mêler de poli-

universel direct en même temps que la Douma d'État, le 12 décembre 1993,

pour deux ans. Il avait donc, à l'époque, une légitimité populaire.

39. Lire notamment MITJUKOV, 2014, pp. 55-56. tique $^{40}$. D'autres pouvoirs ont été supprimés comme celui de se prononcer sur la conformité à la Constitution des actions et des décisions du Président et des autres dirigeants de la Russie et des républiques au sein de la Russie ${ }^{41}$ ou sur la constitutionnalité des partis politiques. Les prérogatives du président de la haute juridiction ont été aussi nettement diminuées afin, comme l'affirmait Nikolaj Vitruk, que celui-ci « ne s'élève plus au-dessus des autres juges mais se contente d'organiser l'activité de la Cour » (Kamyšev, 1994). En particulier, le président a perdu le pouvoir de prendre des mesures d'urgence, comme suspendre l'exécution d'actes attaqués devant la Cour constitutionnelle ${ }^{42}$.

Ces mesures ont-elles permis une dépolitisation de la Cour et un changement de son attitude vis-à-vis du pouvoir exécutif? Vladimir Aleksandrovič Tumanov ${ }^{43}$, qui a été le premier président de la nouvelle Cour, a fait part des craintes qu'il a eues au début de son mandat de voir la Cour être très divisée entre les neuf juges qui s'étaient opposés au décret $n^{\circ} 1400$ durant les événements d'octobre 1993 et les dix autres. Mais il a estimé que ces craintes avaient été excessives ${ }^{44}$. La division entre deux camps antagonistes ne semble pas avoir laissé de traces profondes. Ainsi, lors de l'examen, en 1995, de la fameuse «affaire

40. Voir, sur ce point, l'opinion très tranchée de deux juges de la Cour ayant voté contre la décision du 21 septembre 1993 : l'entretien avec Ernest Ametistov $(V e k, 1994)$ et l'extrait des souvenirs d'Anatolij Kononov (TOROČEŠNIKOVA, 2002). Cette opinion est partagée par les deux anciens présidents de la Cour constitutionnelle, Vladimir Aleksanrovič Tumanov et Marat Viktorovič Baglaj (BAGLAJ, 2001).

41. La Cour avait le pouvoir de se prononcer sur les actions et les décisions de ces dirigeants si, conformément à la Constitution de la Russie, leur inconstitutionnalité pouvait servir de fondement à la destitution de leurs auteurs ou à la mise en œuvre de leur responsabilité (article 74 de la loi du 12 juillet 1991).

42. Ce pouvoir de suspension était prévu à l'article 21.1.6 de la loi sur la Cour constitutionnelle du 12 juillet 1991 (Zakon, 12.07.1991, loc. cit).

43. Vladimir Tumanov (1926-2011), docteur en droit, spécialiste des droits occidentaux (et notamment du droit français), a travaillé à l'Institut de l'État et du droit de l'Académie des sciences de l'URSS puis de la Russie avant de devenir, en 1994, juge à la Cour constitutionnelle de la Fédération de Russie. Élu en 1995 à la tête de la Cour, il a pris sa retraite en 1997.

44. Lire l'entretien de Jean-Pierre Massias avec Vladimir Tumanov dans sa version russe (TUMANOV, 2010). La version française, publiée dans la Revue de justice constitutionnelle est-européenne (MASSIAS, 2002), est loin d'être aussi complète que la version russe sur ce sujet. 
tchétchène », où la Cour a reconnu la conformité à la Constitution de plusieurs actes du Président et du gouvernement décidant de l'intervention militaire en Tchétchénie ${ }^{45}$, les huit opinions dissidentes qui ont été adoptées avaient pour auteurs quatre juges favorables à la décision du 21 septembre $^{46}$ et quatre juges hostiles à cette décision ${ }^{47}$. Les opinions condamnant le plus fermement les actes du Président sont venues de deux juges qui avaient pris parti pour Eltsine en 1993, Anatolij Kononov et Nikolaj Vitruk ${ }^{48}$. Ainsi, les positions ne sont pas restées figées. Signalons à cet égard qu'Anatolij Kononov est l'un des juges de la Cour qui ont rédigé le plus grand nombre d'opinions dissidentes et qu'il a fini par démissionner en 2009 pour protester contre l'emprise croissante de l'exécutif sur la justice en Russie ${ }^{49}$. Il y a donc eu un dépassement de la division entre partisans et adversaires de la décision du 21 septembre, ou, pour simplifier, entre les pro-Parlement et les proPrésident. Ce dépassement a rendu possible l'élection par les juges de la Cour, en 2003, dix ans après les événements d'octobre 1993, de Valerij Zor'kin (qui avait été président de la Cour de 1991 à 1993), au poste de président de la Cour - qu'il occupe encore aujourd'hui. Cette élection, qui a été gagnée de justesse (par 10 voix pour, contre 9 à Marat Baglaj, le président sortant, soutenu par le Kremlin), a surpris à l'époque ${ }^{50}$. Elle

45. Lire notamment, sur cette affaire, qui a donné lieu à de nombreuses études, POMERANZ, 1997; GAZIER, 1999, tout particulièrement p. 1375-1377.

46. Il s'agissait des juges Boris Ebzeev, Gadis Gadžiev, Viktor Lučin et Valerij Zor'kin

47. Il s'agissait des juges Ernest Ametistov, Anatolij Kononov,

Tamara Morščakova et Nikolaj Vitruk.

48. Lire les opinions dissidentes de ces deux juges ainsi que les six autres

et la décision de la Cour en date du 31 juillet 1995 in Sobranie zakonodatel'stva, $\mathrm{n}^{\circ} 33,1995$, art. 3424

49. Lire notamment PUŠKARSKAJA, 2009.

50. Parmi les éléments d'explication de ce résultat inattendu, certains sont relatifs à la composition de la Cour lorsqu'elle a procédé à l'élection de son président le

21 février 2003. Il apparaît, en effet, que cette composition n'était pas exactement la même que lors de la mise en place de la haute juridiction, en 1995. Notamment, la Cour ne comptait plus parmi ses membres trois des quatre juges qui s'étaient opposés à Valerij Zor'kin en 1993 : Ernest Ametistov (décédé en 1998), Tamara Morščakova (à la retraite depuis 2002) et Nikolaj Vitruk (à la retraite le 12 février 2003, quelques jours avant l'élection du président de la Cour). Parmi les juges qui avaient combattu les prises de position de Valerij Zor'kin en 1993, seul

Anatolij Kononov était encore en poste. Plus généralement, à la suite de décès et de départs en retraite, la Cour ne comprenait plus que 7 juges issus de l'ancienne a suscité des espoirs de changement chez certains, des craintes de retour à une justice constitutionnelle politisée chez d'autres. Toutefois, Valerij Zor'kin a vite fait comprendre qu'il avait changé depuis les événements de 1993, qu'il entendait bien rester éloigné de la vie politique et s'en tenir strictement au droit ${ }^{51}$. Ainsi, son élection à la tête de la Cour ne s'est aucunement traduite par un infléchissement de la jurisprudence de la haute juridiction. On constate, depuis que la nouvelle Cour a été mise en place en 1995, une très grande prudence de sa part sur toutes les questions qui concernent de près ou de loin le Président. Est-ce, au moins en partie, dû au « traumatisme » causé par la suspension des activités de la Cour en octobre 1993 et aux menaces qui ont pesé sur son existence? On peut légitimement le supposer.

En guise de conclusion, on se demandera quel regard portent les juges constitutionnels sur ces événements dix ans, voire vingt ans après. Les entretiens accordés dans les années 2000 ou 2010 par les juges ayant pris part à la réunion du 21 septembre 1993 montrent qu'ils sont toujours aussi divisés sur ce que la Cour devait faire ce jour-là. Aucun d'entre eux ne regrette la position qu'il a adoptée. Tous affirment que si c'était à refaire, ils se comporteraient de la même façon. Et c'est vrai aussi bien des juges qui ont voté pour la décision que de ceux qui ont voté contre. Parmi ceux qui ont voté pour, certains, comme Jurij Rudkin, ont un avis très tranché sur la question : « Je ne regrette aucunement les décisions que nous avons prises à ce moment. Elles étaient justes du point de vue de la lettre et de l'esprit de la Constitution [de l'époque] $»^{52}$. D'autres, toutefois, comme Valerij Zor'kin et Gadis Gadžiev, expriment un léger regret. Valerij Zor'kin se demande ainsi si la Cour n'aurait pas dû se tenir à l'écart d'une situation qui échappait au droit $^{53}$. Quant à Gadis Gadžiev, il déplore la précipitation avec laquelle

Cour (contre 13 en 1995). En revanche, elle comportait 12 juges désignés à compter de 1994, lesquels n'avaient donc pas participé à l'activité de la première Cour et aux affrontements qui l'ont marquée. Ces changements ont dû faciliter le retour de V. Zor'kin à la tête de la Cour.

51. Voir les entretiens accordés par Valerij Zor'kin peu de temps après son élection de 2003 à la tête de la Cour constitutionnelle : GAMOV, 2003; SUHOVA \& ZAMAHIN, 2003.

52. Lire l'entretien avec Jurij Rudkin (Rapsinews.ru, 2011)

53. Lire l'entretien précité avec Valerij Zor'kin (SUHOVA \& ZAMAHIN, 2003) 
la décision a été prise ${ }^{54}$. Malgré ces nuances de regret, il apparaît que, même avec le recul, aucun consensus n'émerge sur l'attitude qu'aurait dû adopter la Cour à ce moment.

À défaut d'une opinion commune parmi les juges, existe-t-il une position officielle de la Cour constitutionnelle actuelle sur le sujet? À notre connaissance, il n'y en a pas. En revanche, le site actuel de la Cour n'est pas neutre lorsqu'il rend compte de la décision du 21 septembre 1993. Dans sa rubrique sur l'histoire de la Cour, il l'évoque rapidement en la qualifiant, comme nous l'avons vu, de décision « ayant entraîné les conséquences les plus dramatiques ». Surtout, si l'on consulte les biographies des juges, il apparaît que seules celles consacrées à trois juges ayant voté contre la décision (Ernest Ametistov, Tamara Morščakova et Nikolaj Vitruk) retracent - parfois longuement - leur position à l'égard $\mathrm{du}$ décret $\mathrm{n}^{\circ} 1400$. Uniquement leurs points de vue sont donc donnés sur cette décision historique ${ }^{55}$

\section{RÉFÉRENCES BIBLIOGRAPHIQUES}

AMETISTOV Ernest Mihajlovič (1999-2001), Otjabr'skie vospominanija [Souvenirs d'octobre], Ametistov Foundation 1999-2001; http://www.ametistov.ru/part5.html (consulté le 30.06.2014)

BAGLAJ Marat Viktorovič (2001), « Pervyj Konstitutionnyj Sud byl izlišne politizirovan » [La première Cour constitutionnelle était excessivement politisée], 11.07.2001; http://www.garweb.ru/conf/ ks/20030129/smi/msg.asp@id_msg127767.htm (consulté le 21.06.2014)

BAUDOUIN Marie-Elisabeth (2005), Justice constitutionnelle et État post-soviétique, Clermont-Ferrand : Les Presses universitaires de la Faculté de droit de Clermont-Ferrand; Paris : L.G.D.J., p. 163-214.

BAUDOUIN Marie-Elisabeth (2010), « La Cour constitutionnelle de la Fédération de Russie vue par un juriste français », Les cahiers du Conseil constitutionnel, $\mathrm{n}^{\circ} 28, \mathrm{p}$. 55 et $\mathrm{s}$.

54. Lire l'entretien précité avec Gadis Gadžiev (VIŠNEVSKIJ, 2011).

55. Voir le site précité de la Cour.
EPSTEIN Lee, KNIGHT Jack \& SHEVTSOVA Olga (2001), « The Role of Constitutional Courts in the Establishment and Maintenance of Democratic Systems of Governement », Law and Society Review, vol. $35, n^{\circ} 1$, p. $117-163$.

GAMOV Aleksandr (2003), « Ja ne "revanšist". Revoljucij v sude ne budet » [Je ne suis pas « revanchard ». Il n'y aura pas de révolution à la Cour], entretien avec Valerij Zor'kin, Komsomol'skaja Pravda, 03.03.2003.

GAZIER Anne (1999), « Justice constitutionnelle et fédéralisme en Russie », Revue du droit public, $n^{\circ} 5$.

GÉLARD Patrice (1993), « L'actualité constitutionnelle en Russie et dans les pays de l'Europe de l'Est et de la CEI (août-octobre $1993) »$, Revue française de droit constitutionnel, $\mathrm{n}^{\circ} 16, \mathrm{p} .857$ et $\mathrm{s}$.

KAMYŠEV Dmitrij (1994), « Konstitucionnyj sud stanet bolee nezavisimym » [La Cour constitutionnelle deviendra plus indépendante], Gazeta Kommersant”, 03.02.1994; http://www.kommersant.ru/doc/70277 (consulté le 25.08.2016).

Konstitucionnyj Sud. Epoha Zor'kina (oktjabr' 1991-oktjabr' 1993) [La Cour constitutionnelle. L'époque de Zor'kin (octobre 1991-octobre 1993)]; http://1993.sovnarkom.ru/TEXT/STATYI/ks_91_93.htm (consulté le 25.08.2015).

MASSIAS Jean-Pierre (2002), « Entretien avec Vladimir Alexandrovitch Toumanov », Revue de justice constitutionnelle est-européenne (n 3, 2002, p. 337-342); http://www.est-europa.univ-pau.fr/images/ archives/2002-2008/2002_3.pdf (consulté le 25.08.2016).

MITJUKOV M. A. (2014), Roždenie Konstitucii Rossii. Konstitucionnoe soveščanie 1993 goda [La naissance de la Constitution de la Russie. L'Assemblée constituante de 1993], M. : Prospekt.

Ogonëk (2005), « Ot avtorov » [De la part des auteurs], Ogonëk, $\mathrm{n}^{\circ} 43$, 24-30 octobre 2005; http://www.ogoniok.com/4917/8/ (consulté le 20.06.2014).

Političeskij Žurnal (2004), "S nami nikto ne smel tak obščat'sja » [Personne n'a osé nous parler ainsi], entretien avec Nikolaj Vedernikov, Političeskij Žurnal, 15.11.2004.

POMERANZ William E. (1997), « Judicial Review and the Russian Constitutional Court : the Chechen Case », Review of Central and EastEuropean Law, ${ }^{\circ}$ 1, p. 9-48 (DOI: 10.1163/157303597124666375). 
PUSKARSKAJA Anna, « Konstitucionnyj sud terjaet osobye mnenija » [La Cour constitutionnelle perd des opinions dissidentes], Gazeta Kommersant", 02.12.2009; http://www.kommersant.ru/doc/1284828 (consulté le 25.08.2016).

Rapsinews.ru (2011), « Sud'ja KS RF Jurij Rudkin : V oktjabre 1993 goda sud vse delal po zakonu » [Le juge de la CC de la FR Jurij Rudkin : en octobre 1993, la Cour a agi entièrement conformément à la loi], 28.10.2011;

http://www.rapsinews.ru/judicial analyst/20111028/256786826.html (consulté le 26.08.2016).

SUHOVA Svetlana \& ZAMAHIN Andrej (2003), « Konstitucija i revoljucija » [La Constitution et la révolution], entretien avec Valerij Zor'kin, Itogi, $\mathrm{n}^{\circ}$ 9, 06.03.2003 $\left(\mathrm{n}^{\circ} 351\right)$.

TOROČEŠNIKOVA Mar'janna (2002), Konstitutucija [La Constitution], Radio Svoboda, 09.12.2002, et notamment l'extrait des souvenirs d'Ernest Ametistov :

http://svoboda.org/articleprintview/24199328.html (consulté le 01.07.2014).

TROCHEV Alexei (2008), Judging Russia : The Role of the Constitutional Court in Russian Politics 1990-2006 (chapitres 3, 4, 5), Cambridge :

Cambridge University Press.

TUMANOV Vladimir (2010), Vladimir Aleksandrovič Tumanov. Izbrannoe [Vladimir Aleksandrovič Tumanov. Euvres choisies], Moscou, Norma, Infra-M, 2010, p. 498

Vek (1994), « Konstitucionnyj sud ne vyše vseh sudov » [La Cour constitutionnelle n'est pas supérieure à toutes les autres juridictions], entretien avec Ernest Ametistov, Vek, n 38 (107), 7-13.10.1994 : $\mathrm{http}: / /$ www.terralegis.org/terra/amet/3amet14.html (consulté le 21.06.2014).

VIŠNEVSKIJ Boris (2011), « Sud'ja Gadis Gadžiev : "My sobralis' večerom, dejstvitel'no, bez mantij" » [Le juge Gadis Gadžiev : « Nous nous sommes réunis le soir, pour de vrai, sans robe »], entretien avec Gadis Gadžiev, Novaja Gazeta, 16.06.2011; http://www.novayagazeta.ru/politics/47691.html (consulté le 26.08.2016).

VITRUK N. V. (2001), Konstitucionnoe pravosudie v Rossii (1991-2001 gg.) : očerki teorii i praktiki [La justice constitutionnelle en Russie (1991-2001) : essai de théorie et de pratique], M. : Gorodec-Izdat, 2001 (de larges extraits de cet ouvrage ont été publiés sur le site internet : swotanaliz.ru).
ZAKATNOVA, Anna (2001), « Sud'ja Gadis Gadžiev : Konstitucionnyj sud dolžen byt' nekim protivovesom demokratii » [Le juge Gadis Gadžiev : la Cour constitutionnelle doit être une sorte de contrepoids à la démocratie], Nezavisimaja Gazeta, 02.11.2001; http://www.ng.ru/politics/2001-11-02/2 gadzhiev.html (consulté le 23.08.2016).

\section{SOURCES JURIDIQUES}

\subsection{Avis et déclarations}

Commission de Venise (1994), Avis sur la Constitution de la Fédération de Russie adoptée en votation populaire le 12 décembre 1993, CDL(1994)011f-restr, Strasbourg, 16 mars 1994.

Zajavlenie Predsedatelja Konstitutionnogo Suda Rossijskoj Federacii ot 22 sentjabrja $1993 \mathrm{~g}$.

« Konstitucionnyj krizis et vozmožnye mery po ego preodoleniju »

Déclaration du président de la Cour constitutionnelle de la Fédération de Russie « La crise constitutionnelle et les mesures permettant de la surmonter »], Vedomosti S"ezda Narodnyh Deputatov Rossijskoj Federacii i Verhovnogo Soveta Rossijskoj Federacii (VSndRF i VSRF), $\mathrm{n}^{\circ} 39$, du 6 octobre 1993 (reconstitué), art. 1702; http://constitution. garant.ru/files/961/39.pdf (consulté le 8 juillet 2014).

Trebovanie Predsedatelja Konstitutionnogo Suda Rossijskoj Federacii ot 28 sentjabrja $1993 \mathrm{~g}$.

[Demande du président de la Cour constitutionnelle de la Fédération de Russie], VSndRF $i$ VSRF, n 39 du 6 octobre 1993 (reconstitué), art. 1706; http://constitution.garant.ru/files/961/39.pdf (consulté le 8.07.2014)

Zajavlenie Konstitucionnogo Suda Rossijskoj Federacii ot 5 okrjabrja $1993 \mathrm{~g}$.

[Déclaration de la Cour constitutionnelle de la Fédération de Russie], 5 octobre 1993, VSndRF $i$ VSRF, n 39 du 6 octobre 1993 (reconstitué), art. 1708 .

\subsection{Législation}

Zakon RSFSR ot 12 ijulja $1991 \mathrm{~g}$.

«O Konstitucionnom Sude RSFSR » [Loi de la RSFSR du 12 juillet 1991 «Sur la Cour constitutionnelle de la RSFSR »], Vedomosti S"ezda Narodnyh Deputatov RSFSR $i$ Verhovnogo Soveta RSFSR, $1991, n^{\circ} 30$, art. 1017 
Ukaz Prezidenta Rossijskoj Federacii ot 20 marta 1993 g. «O dejatel'nosti ispolnitel'nyh organov do preodolenija krizisa vlasti » n 379 [Décret du Président de la Fédération de Russie « sur l'activité des organes exécutifs jusqu'à ce que la crise du pouvoir soi surmontée », en date du 20 mars 1993], Sobranie aktov Prezidenta $i$ Pravitel'stva $R F, 1993, \mathrm{n}^{\circ} 13$, art. 1102.

Ukaz Prezidenta Rossijskoj Federacii ot 21 sentjabrja 1993 g. $\mathrm{n}^{\circ} 1400$ «O poetapnom konstitucionnom reforme v Rossijskoj Federacii » [décret du Président de la Fédération de Russie "Sur la réforme constitutionnelle par étapes dans la Fédération de Russie"]; http://kremlin.ru/acts/bank/4364 (consulté le 22.08.2016).

Ukaz Prezidenta Rossijskoj Federacii ot 7 oktjabrja

«O Konstitucionnom Sude Rossijskoj Federacii » [Décret du Président de la Fédération de Russie du 7 octobre 1993 « Sur la Cour constitutionnelle de la Fédération de Russie »], Sobranie aktov Prezidenta i Pravitel'stva, 1993, n 41, art. 3929.

\subsection{Jurisprudence}

Postanovlenie Konstitucionnogo Suda Rossijskoj Federacii ot 14 janvarja $1992 \mathrm{~g}$.

« Po delu o proverke konstitucionnosti Ukaza Prezidenta RSFSR ot 19 dekabrja 1991 goda "Ob obrazovanii Ministerstva bezopasnost i vnutrennih del RSFSR" » [Arrêt de la Cour constitutionnelle de la Fédération de Russie « Sur l'examen de la constitutionnalité du décret du président de la RSFSR du 19 décembre 1991 "Sur la création du ministère de la Sécurité et de l'Intérieur de la RSFSR" »], n 1-P, 14 janvier 1992, Vestnik Konstitutionnogo Suda Rossijskoj Federacii, $\mathrm{n}^{\circ} 1,1993$.

Postanovlenie Konstitucionnogo Suda Rossijskoj Federacii ot 30 nojabrja $1992 \mathrm{~g}$.

"Po delu o proverke konstitucionnosti Ukazov Prezidenta Rossijskoj Federacii ot 23 avgusta 1991 g. $n^{\circ} 79$ "O priostanovlenii dejatel'nosti kommunističeskoj partii RSFSR”, ot 25 avgusta 1991g. n 90 "ob imuščestve KPSS i kommunističeskoj partii RSFSR" i ot 6 nojabrja 1991 g. n 169 "O dejatel'nosti KPSS I KP RSFSR", a takže o proverke konstitutionnosti KPSS I KP RSFSR » [Arrêt de la Cour constitutionnelle de la Fédération de Russie en date du 30 novembre 1992 «Sur l'examen de la constitutionnalité des décrets du président de la Fédération de Russie du 23 août 1991, $n^{\circ} 79$ "Sur la suspension de l'activité du Parti communiste de la RSFSR", du 25 août 1991, n 90, "Sur le patrimoine du PCUS et du Parti communiste de la RSFSR”, et du 6 novembre 1991, n 169 ,
"Sur l'activité du PCUS et du PC de la FSFSR" ainsi que sur l'examen de la constitutionnalité du PCUS et du PC de la RSFSR »], n 9-P, Sobranie zakonodatel'stva Rossijskoj Federacii, 1993, n 11, art. 400.

Postanovlenie Konstitucionnogo Suda Rossijskoj Federacii ot 12 fevralja $1993 \mathrm{~g}$.

« Po delu o proverke konstitutionnosti Ukaza Prezidenta Rossijskoj Federacii ot 28 oktjabrja 1992 goda "O merah po zaščite konstitucionnogo stroja Rossijskoj Federacii” " [Arrêt de la Cour constitutionnelle de la Fédération de Russie « Sur l'examen de la constitutionnalité du décret du Président de la Fédération de Russie du 28 octobre 1992 "Sur les mesures destinées à défendre l'ordre constitutionnel de la Fédération de Russie" »] n 3-P, 12 février 1993 Vestnik Konstitucionnogo Suda Rossijskoj Federacii, n 1, 1994

Zaključenie Konstitucionnogo Suda Rossijskoj Federacii ot 23 marta $1993 \mathrm{~g}$.

«O sootvetstvii Konstitucii Rossijskoj Federacii dejstvij i rešenij Prezidenta Rossijskoj Federacii B.N. El'cina, svjazannyh s ego Obraščeniem k graždanam Rossii 20 marta 1993 goda » [Décision de la Cour constitutionnelle de la Fédération de Russie en date du 23 mars 1993 « Sur la conformité à la Constitution de la Fédération de Russie des agissements et des décisions adoptés en lien avec sa Déclaration aux citoyens de la Russie du 20 mars $1993 »]$; http://www.szrf.ru/doc. phtml?nb=edition 10\&issid $=1993013000 \&$ docid $=116$

Postanovlenie Konstitucionnogo suda Rossijskoj Federacil ot 21 aprelja $1993 \mathrm{~g}$.

« Po delu o proverke konstitucionnosti časti vtoroj punkta 2 postanovlenija S"ezda narodnyh deputatov Rossijskoj Federacii ot 29 marta 1993 "O vserossijskom referendume 25 aprelja 1993, porjadke podvedenija ego itogov i mehanizme realizacii rezul'tatov referenduma" » [Arrêt de la Cour constitutionnelle de la Fédération de Russie en date du 21 avril 1993 « sur l'examen de la constitutionnalité de la deuxième partie du point 2 de l'arrêté du Congrès des députés du peuple de la Fédération de Russie du 29 mars 1993 "Sur le référendum panrusse du 25 avril 1993, sur le mode d'établissement de ses résultats et les modalités de leur mise en œuvre" »], n 8-P, Vestnik Konstitucionnogo Suda Rossijskoj Federacii, n 2-3, 1994.

Zaključenie Konstitucionnogo Suda Rossijskoj Federaci ot 21 sentjabrja $1993 \mathrm{~g}$.

«O sootvetstvii Konstitucii Rossijskoj Federacii dejstvij i rešenij Prezidenta Rossijskoj Federacii, svjazannyh s ego ukazom "O poetapnoj konstitucionnom reforme v Rossijskoj Federacii" 
ot 21 sentjabrja 1993 goda $n^{\circ} 1400$ i obraščeniem k graždanam Rossii 21 sentjabrja 1993 goda » [Décision de la Cour constitutionnelle de la Fédération de Russie en date du 21 septembre 1993 «Sur la conformité à la Constitution de la Fédération de Russie des agissements et des décisions adoptés par le président de la Fédération de Russie en lien avec son décret "Sur la réforme constitutionnelle par étapes dans la Fédération de Russie" du 21 septembre 1993 n 1400 et avec sa déclaration aux citoyens de la Russie du 21 septembre $1993 »]$, Vestnik Konstitutionnogo Suda Rossijskoj Federacii, n 6, 1994. 